\title{
A Multi-modal Data Merging Framework for Correlative Investigation of Strain Localization in Three Dimensions
}

\author{
M.A. CHARPAGNE (10, ${ }^{1,2,4,5}$ J.C. STINVILLE, ${ }^{1,2}$ A.T. POLONSKY, ${ }^{1,3}$ \\ M.P. ECHLIN, ${ }^{1}$ and T.M. POLLOCK ${ }^{1}$ \\ 1.-Materials Department, University of California Santa \\ Barbara, Santa Barbara, CA 93106, USA. 2.-Present address: Department of Materials Science \\ and Engineering, University of Illinois at Urbana-Champaign, Urbana, IL 61801, USA. \\ 3.-Materials Mechanics \& Tribology Department, Sandia National \\ Laboratories, Albuquerque, NM 87185, USA. 4.—e-mail: mcharpagne@ucsb.edu. \\ 5.—e-mail: mcharp@illinois.edu
}

\begin{abstract}
A multi-modal data-merging framework that enables the reconstruction of slip bands in three dimensions over millimeter-scale fields of view is presented. The technique combines 3D electron back-scattered diffraction (EBSD) measurements with high-resolution digital image correlation (HR-DIC) information collected in the scanning electron microscope (SEM). A typical merging workflow involves the segmentation of features within the strain field (slip bands, deformation twins) and the microstructure (grains), alignment of datasets and the projection of slip bands into the 3D microstructure, using the knowledge of the local crystallographic orientation. This method is demonstrated in two materials: a face-centered cubic (FCC) nickel-base superalloy and hexagonal close-packed (HCP) titanium alloy.
\end{abstract}

\section{INTRODUCTION}

Prediction of macroscopic mechanical behavior requires a detailed understanding of the micromechanical response at the grain or sub-grain scale, which is often highly heterogeneous. Heterogeneities such as slip bands, deformation twins, kink bands and grain boundary sliding may dominate the constitutive response of a material under a variety of loading conditions. Studies of these deformation modes usually involve the mapping of the 2D microstructure, generally utilizing electron back-scattered diffraction (EBSD), alongside measurements of slip or twinning traces collected using secondary electron images, backscattered electron images, electron channeling contract imaging collected in the scanning electron microscope (SEM), high-resolution digital image correlation (HR-DIC) or atomic force miscroscopy. ${ }^{1-17}$ While general trends can be extracted from these combined measurements, they do not enable examination of the

(Received June 3, 2021; accepted August 31, 2021;

published online September 27, 2021) origin of deformation events such as slip bands within a given microstructure. For instance, slip traces observed on a specimen's free surface are a projection of a three-dimensional strain localization event that often initiates within the depth of the material. Therefore, a better understanding of strain localization phenomena would involve the correlative mapping, not in $2 \mathrm{D}$, but of the $3 \mathrm{D}$ material microstructure. In this context, the present article focuses on the combination of slip trace analyses with $3 \mathrm{D}$ microstructure data over large regions of interest (ROIs).

HR-DIC techniques have been developed and extended for use with measurements collected in the SEM, 8,18 where the nanometer-scaled spatial imaging resolution enables early detection of individual slip bands on the specimen surface. ${ }^{19}$ Recent developments in SEM imaging and accompanying software have enabled the collection of large data sets over wide fields of view, with increasingly high spatial resolution and richness of information. Using microscope automation algorithms, these high-resolution strain measurements are mapped over $\mathrm{mm}^{2}$-scaled areas, easily capturing the information over statistically relevant numbers of 
grains..$^{20,21}$ In parallel, robust 3D microstructure characterization tools have emerged in the past decade, including non-destructive techniques such as high-energy x-ray diffraction using synchrotron sources, ${ }^{22,23}$ laboratory-scale diffraction contrast tomography (lab-DCT) and ablation techniques such as EBSD tomography using mechanical polishing (i.e., the Robo-Met system) ${ }^{24,25}$ plasma focused ion beams (FIBs) or femtosecond lasers, which involve serial sectioning of the material and data collection on consecutive slices to reconstruct the 3D microstructure. ${ }^{26}$ These hardware and software advances have greatly reduced data collection time such that the most time-consuming step is often data analysis. In this context, the aim of the present article is to introduce a multi-modal data-merging technique that enables automated mapping of the micro-mechanical strain field as a function of microstructure and extraction of non-biased correlative statistics. This method is demonstrated here for the merging of DIC and 3D EBSD data, but can be applied to other types of multi-modal datasets. The following sections discuss the topics of data collection, alignment and merging through two illustrative examples that show slip in a nickelbase superalloy and a titanium alloy.

\section{MULTI-MODAL DATA COLLECTION}

Gathering multi-modal data often requires specifically designed or prepared samples that satisfy the requirements of the constituent experimental techniques. The multi-modal datasets shown presently use in situ mechanically loaded samples that permit the collection of HR-DIC strain measurements and 2D EBSD from an external surface in the gauge section as well as a 3D TriBeam dataset from the same region. A schematic of a sample used for this multi-modal data collection is shown in Fig. 1. One face of the gauge of the specimen has strain measured by HR-DIC (xz); it is colored in teal in the figure. This face was mirror polished and prepared as detailed in the next section. A speckle adapted to HR-DIC is then deposited onto the surface. DIC measurements are carried out in situ using a custom-built tensile stage. The specimen is then un-mounted from the stage, and a pedestalshaped sample is extracted out from the gauge section, around the ROI, following the blue line shown in the figure. The DIC surface is then protected with silver paint, and the sample is loaded back in the SEM chamber for Tribeam tomography from the ROI. The femtosecond laser is used to iteratively ablate layers from the sample pedestal along the z-direction, with the incident beam parallel to the opposite surface of the DIC measurement surface. (a)

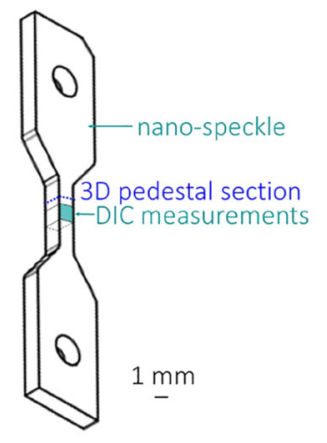

(b)

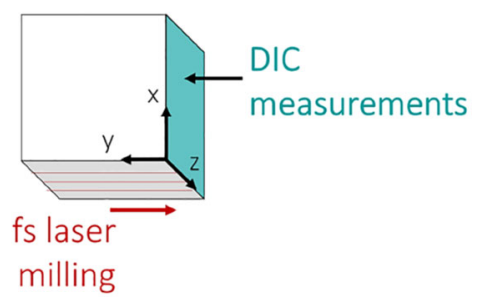

Fig. 1. Specimen geometry and location of the multi-modal measurements: (a) overview; (b) region of interest.

\section{High-Resolution Digital Image Correlation (HR-DIC)}

Prior to mechanical testing, Ti7-Al ${ }^{27}$ and wrought Inconel 718 nickel-base superalloy ${ }^{28}$ specimens were polished with $\mathrm{SiC}$ papers up to 1200 grit, followed by polishing with a 6 - $\mu \mathrm{m}$ diamond suspension and then chemo-mechanical polishing with $0.05-\mu \mathrm{m}$ colloidal silica for $12 \mathrm{~h}$. A nanoscale speckle pattern for HR-DIC measurements, with an average particle size of $60 \mathrm{~nm}$, was produced using the gold nanoparticle speckle deposition patterning technique developed by Kammers et al. ${ }^{29}$ SEM image sets were acquired before loading and after unload from $1 \%$ total deformation, following the procedures developed by Kammers and Daly ${ }^{29}$ and Stinville et al. ${ }^{8}$ A National Instruments ${ }^{\text {TM }}$ scan controller and acquisition system (DAQ) were used for control beam scanning in a modified Thermo Fisher Scientific Dual Beam FIB microscope. ${ }^{30}$ This custom beam scanner removes the SEM beam defects associated with some microscope scan generators and reduces drift distortions. ${ }^{31}$ Tiles of $6 \times 4$ images for the titanium alloy and $8 \times 8$ images for the nickel-base superalloy are acquired before and after deformation, with an image overlap of $15 \%$. DIC calculations are performed on these series of images, and the tiles are merged using a pixel resolution merging procedure. Regions of about $0.9 \times 0.3 \mathrm{~mm}^{2}$ and $1 \times 1 \mathrm{~mm}^{2}$ were investigated for the titanium alloy and nickel-base superalloy, respectively. Subset sizes of $31 \times 31$ pixels $(1044 \mathrm{~nm} \times 1044 \mathrm{~nm})$ with a step size of 3 pixels $(101 \mathrm{~nm})$ were used for DIC measurements. Digital image correlation was performed using the Heaviside-DIC method ${ }^{32}$ to quantitatively characterize 
the amplitude of slip localization. The sample preparation, imaging conditions and HeavisideDIC parameters allow for a discontinuity detection resolution between 0.2 and 0.3 pixels (offsets induced by slip of $7 \mathrm{~nm}$ and $10 \mathrm{~nm}$, respectively). ${ }^{32}$ Examples of resulting $\epsilon_{x x}$ strain maps ( $x$ being the loading direction) are displayed in Figs. $4 \mathrm{~b}$ and 6c.

\section{Three-dimensional EBSD}

The recently commercialized TriBeam system, ${ }^{30,33}$ which combines a femtosecond laser with a focused ion beam (FIB-SEM), is used to ablate micron-thick layers of material over $\mathrm{mm}^{2}$ areas. During a serial sectioning experiment, at each slice, images are captured using backscatter and secondary electron detectors as well as the mapping of grain orientations using EBSD. EBSD can be collected with sub-micron spatial resolution and crystallographic orientation indexing accuracy sufficient to capture subgrain orientation gradients. The indexing accuracy can be further enhanced by collecting and storing raw EBSD patterns, which are later re-indexed using the EMSphInx spherical indexing software. ${ }^{34}$ Datasets with volumes approaching $1 \mathrm{~mm}^{3}$ with cubic-micron sized voxels are now readily obtainable, ${ }^{26}$ with collection times on the order of days to a week, depending on the data modalities and voxel resolution. The sections are then stacked back together using the conventional filters with the DREAM.3D software. ${ }^{35}$

As the barriers for gathering large 3D multimodal datasets are reduced, this motivates efforts on accurate reconstruction, analysis and interpretation of the large datasets, which may contain as much as 1 TB of information.

\section{MERGING WORKFLOW}

\section{Correction of Spatial Distortions}

Among the collected datasets, 3D EBSD suffers from the highest spatial distortions as a result of the large fields of view, large sample tilt required for EBSD (and the associated geometric distortions) and beam drift. ${ }^{12,36}$ These effects directly alter the shape of the grains, as illustrated in Fig. 2a,b. Therefore, it is critical to compensate for these distortions before merging the DIC data. BSE images are collected at $0^{\circ}$ tilt along with EBSD data on every slice, as shown in Fig. 2c. These images are collected with much shorter exposure times per pixel compared to EBSD and exhibit reduced distortion. As the BSE images reveal some crystallographic contrast, they can be used as a reference for the correction of EBSD data. The relative distortion between BSE and EBSD data of a given slice can be modeled with a $2 \mathrm{D}$ polynomial function of degree 3 using the formula shown in Eq. 1. To register the function and find its $c_{i, j}^{x}$ coefficients, either manually selected control points are used or the automatic registration method described in Ref. 12 is invoked, using segmented grain boundaries from both the EBSD and BSE data as fiducials for alignment. Calibration of the distortion is performed every 50 slices throughout the dataset. If its coefficients remain constant, a unique set of coefficients is used to align the whole dataset. This was the case for both datasets presented in this article. The aligned EBSD map corresponding to Fig. 2a is shown in b. The dataset section is now square, similar in appearance to the BSE image, and the DIC surface is now planar.

$$
f(x, y)=\left\{\begin{array}{l}
x^{\prime}=\sum_{n=0}^{3} \sum_{k=0}^{n} c_{k, n-k}^{x} x^{k} y^{n-k} \\
y^{\prime}=\sum_{n=0}^{3} \sum_{k=0}^{n} c_{k, n-k}^{y} x^{k} y^{n-k}
\end{array}\right.
$$

At this stage, the dataset is aligned in the $\mathrm{XY}$ plane, and the DIC data are ready to be aligned on the XZ surface. To do so, the alignment procedure described above is applied to align the DIC data onto the 3D EBSD dataset and a second distortion function $f(x, z)$ is calibrated using pairs of control points.

\section{Feature Segmentation}

Individual slip traces are segmented from the DIC maps using conventional image processing techniques detailed in Ref. 28 and assigned a number ID. It is worth mentioning that this step could benefit from more advanced vectorization algorithms involving computer vision, for example. ${ }^{37}$ Accurate segmentation enables detection of the exact location of the slip traces in the HR-DIC reference frame. In particular, the locations of the end points of each slip trace are listed. On the other hand, individual grains are segmented from the 3D EBSD dataset using the filters that are readily available in the DREAM.3D software.

\section{Three-dimensional Reconstruction of Slip Bands}

Once the DIC slip traces have been segmented, their coordinates in the reference frame of the EBSD dataset are calculated using the distortion function $f(x, z)$. The local crystallographic orientation associated with every slip band is then known. The angles $(\phi 1, \Phi, \phi 2)$ are the average Euler angles of the local grain associated to a given slip band. As a limited set of slip planes are available (e.g., 111 planes in a FCC lattice), the angle of the slip trace on the DIC surface can be compared to the traces of the theoretical planes. If a candidate plane of Miller indices $(h, k, l)$ exhibits a trace that deviates by $2^{\circ}$ or less from that of the slip band, it is assigned to the slip band. Given its indices, the normal to the slip plane in the reference frame of the crystal can be calculated, defining the normal vector to the plane $\mathbf{n}_{\text {crystal }}$. The plane normal in the sample's reference frame is obtained by rotating it according 

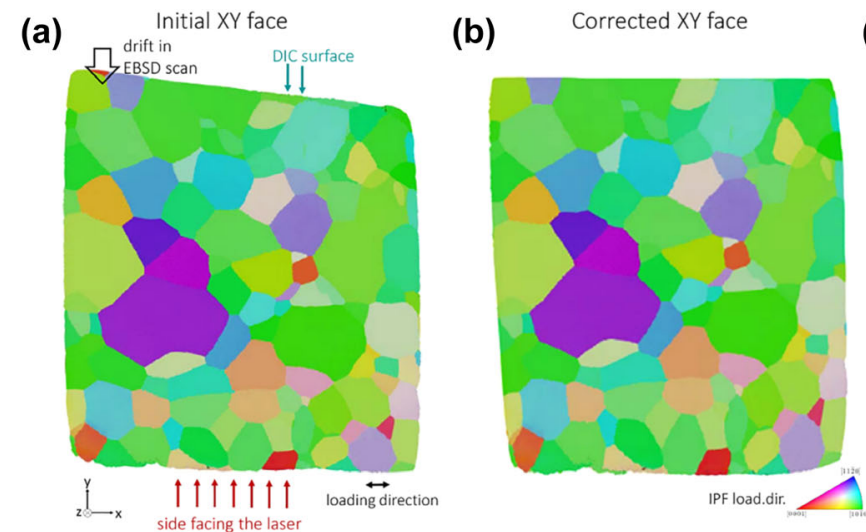

(c) Corresponding BSE image

Fig. 2. Compensation of spatial distortions in 3D EBSD datasets using multi-modal data merging. (a) As-collected XY slice, (b) corrected XY slice, aligned onto (c) the corresponding BSE image.

to Eq. 2, where the rotation matrix $\mathbf{R}_{\text {sample,crystal }}$ is defined as in Eq. 3.

$$
\mathbf{n}_{\text {sample }}=\mathbf{R}_{\text {sample,crystal }} \cdot \mathbf{n}_{\text {crystal }}
$$

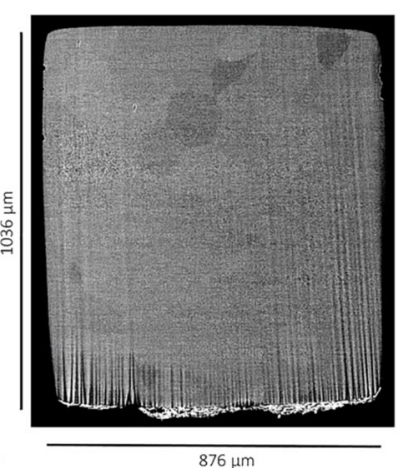

rotation matrix to create the rendering shown in Fig. 3b. Another example is shown in Fig. 3c and d in an HCP grain favorably oriented for prismatic slip. The (1100) slip traces are shown in the 3D

$$
\left(\begin{array}{ccc}
\cos \phi_{1} \cos \phi_{2}-\sin \phi_{1} \sin \phi_{2} \cos \Phi & -\cos \phi_{1} \sin \phi_{2}-\sin \phi_{1} \cos \phi_{2} \cos \Phi & \sin \phi_{1} \sin \Phi \\
\sin \phi_{1} \cos \phi_{2}+\cos \phi_{1} \sin \phi_{2} \cos \Phi & -\sin \phi_{1} \sin \phi_{2}+\cos \phi_{1} \cos \phi_{2} \cos \Phi & -\cos \phi_{1} \sin \Phi \\
\sin \phi_{2} \sin \Phi & \cos \phi_{2} \sin \Phi & \cos \Phi
\end{array}\right)
$$

Two renderings are used for visualization: the first one consists of creating slip bands as voxelized objects while the second consists of creating the slip band as an object in the Paraview software. ${ }^{38}$ In the first case, the voxels that belong to a slip band are readily assigned and the DREAM.3D file is edited so that is contains a new entry dedicated to the visualization of slip bands according to their number ID. Using this method, when two slip bands are closer to one another by less than a voxel, they become merged together. The second visualization method involves creating a 'slice' object within the grain of interest in Paraview, using the coordinates of one point on the slip band and its normal vector. This method does not induce any merging of the slip bands. Two examples are shown in Fig. 3 utilizing the second method. The Paraview software is used for data visualization. Figure 3 shows the reconstruction of a (111) slip band in a FCC crystal. The slip trace of interest is highlighted with a red box in Fig. 3a, which is a composite image of IPF colored EBSD (with reference to the loading direction) with the slip traces segmented from the DIC in overlay. Comparison with the traces of all 111 planes reveals that the slip plane is $(1 \overline{1} 1)$. This plane is then rotated according to the grain's (a)

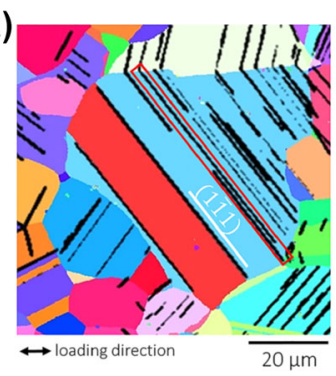

(c)

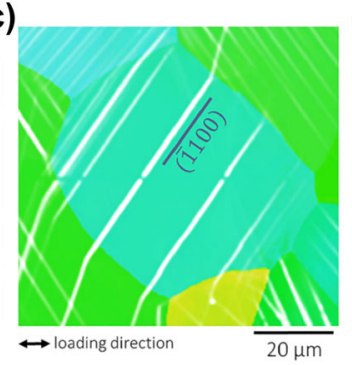

(b)

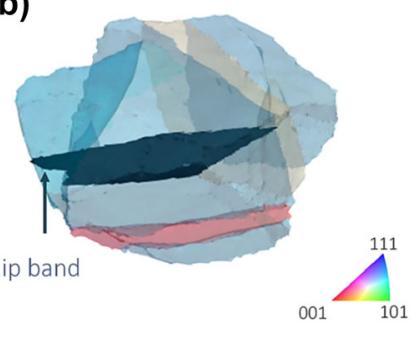

(d)

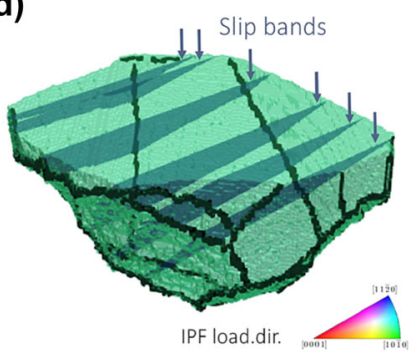

Fig. 3. Three-dimensional representation of the slip bands in Inconel $718(a-b)$ and Ti7Al (c-d). (a) Segmented slip traces obtained from HR-DIC overlapped with the aligned IPF colored EBSD map. The active plane associated to the slip band highlighted with a red box is (111); (b) corresponding projection of the slip band into the 3D microstructure; (c) slip traces obtained from HR-DIC overlapped with the IPF colored EBSD map of the Ti7Al material and showing prismatic slip on (1100); (d) projected slip bands into the 3D microstructure (Color figure online). 
rendering in Fig. 3d. This procedure, involving the detection and assignment of the crystallographic slip plane associated with every slip band within a given dataset, as well as the assignment of the slip band voxels, is fully automated using a custom Python code. This operation is repeated over hundreds of slip bands, which a dataset of millimeter scale typically contains. The next section illustrates application of this method in two different case studies.

\section{CASE STUDIES}

Two high-strength structural materials have been studied: wrought Inconel 718 and titanium Ti-7Al. Unique characteristics of plastic deformation in these FCC and HCP materials, respectively, are revealed in the following sections.

\section{Incipient Plasticity in a Wrought Polycrystalline Nickel-base Superalloy}

A sample of wrought annealed Inconel 718 was deformed monotonically in tension at room temperature, in situ in the SEM. DIC data were collected at the macroscopic yield point of the material $(0.2 \%$ plastic strain). The engineering stress-strain curve is shown in Fig. 4a. The strain field measured by HR-DIC over the ROI at macroscopic yield is shown in Fig. 4. Hundreds of slip bands of various intensity are visible on the free surface of the specimen. A 3D EBSD-DIC dataset of dimensions $549 \mu \mathrm{m} \times 526 \mu \mathrm{m}$ $\times 420 \mu \mathrm{m}$ was collected, as shown in Fig. $4 \mathrm{c}$. A total of 570 slip bands were segmented from HR-DIC data and added to the DREAM.3D dataset for statistical analysis.

The merged dataset is shown in Fig. 5a, where the slip bands are overlaid on the IPF colored EBSD dataset (with respect to the loading direction). A systematic investigation of the location of every slip band in relation to the microstructure reported elsewhere $^{39}$ revealed strong correlations between the location of the most intense slip bands and some annealing twin boundaries ( $\Sigma 3$ boundaries, defined as $60^{\circ}$ rotations around a $<111>$ direction). This configuration is referred to as a parallel slip configuration. ${ }^{8,19}$ In this configuration, slip bands preferentially form close and parallel to particular twin boundaries that exhibit a high difference in directional elastic modulus or a high Schmid factor. Whereas this configuration had already been reported using $2 \mathrm{D}$ measurements, ${ }^{8}$ the investigation of slip band morphology in the 3D microstructure further revealed a strong correlation between the locations of slip bands and triple junctions. A typical example is presented in Fig. 5b, where a slip band (shown in dark blue) emanates from the triple junction formed by the three IPF colored grains, shown in transparency. The free surface where the HR-DIC measurements were conducted is the flat surface on the top. As two of the three grains that
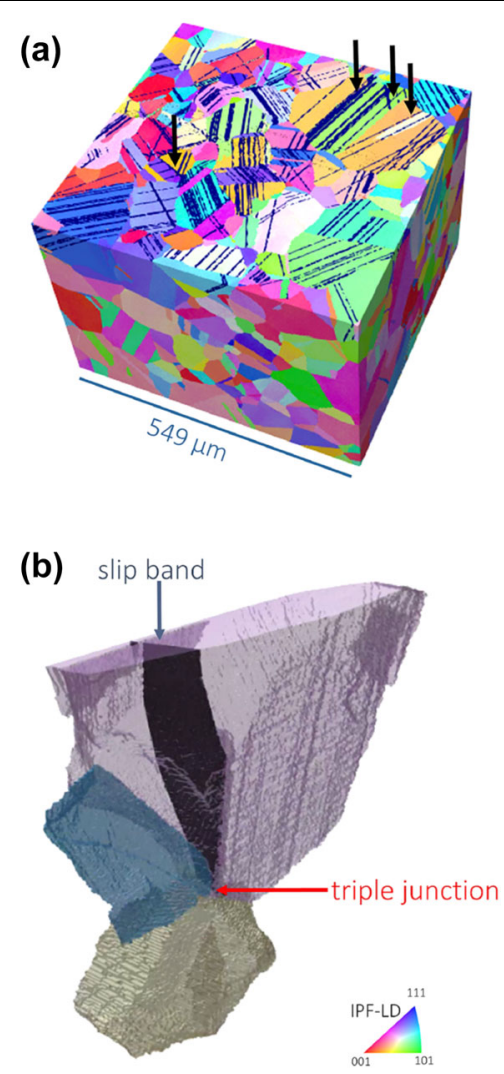

Fig. 5. Merged Inconel 718 dataset: (a) slip bands (in blue) overlaid on the IPF colored 3D EBSD dataset; black arrows indicate parallel slip configurations; (b) example of a slip band emanating from a triple junction (Color figure online).

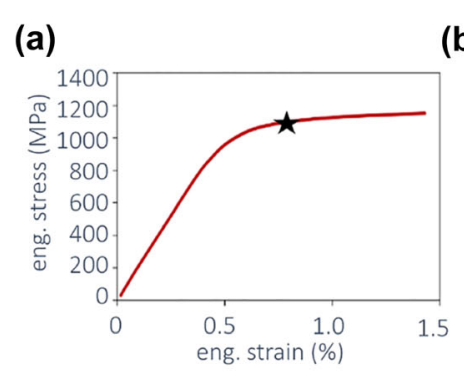

(b)

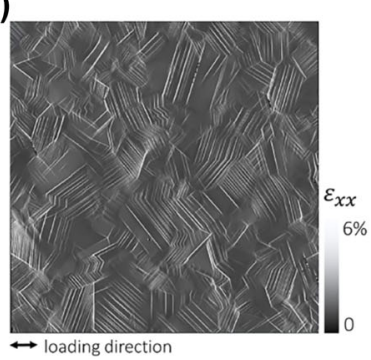

(c)

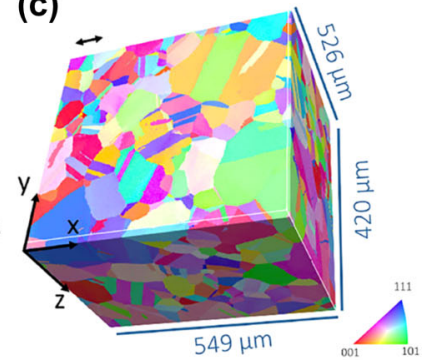

Fig. 4. Inconel 718 3D EBSD-DIC dataset: (a) engineering stress-strain curve, (b) strain field of the ROI obtained from HR-DIC, (c) 3D EBSD dataset (Color figure online). 
define the triple junction are fully contained in the bulk of the material (not visible on the free surface), this configuration could not have been detected from $2 \mathrm{D}$ measurements only. This kind of configuration is a good example of the importance and relevance of capturing the $3 \mathrm{D}$ microstructure. In this dataset, over two thirds of the slip bands are connected to triple junctions. ${ }^{39}$

In conclusion, strong correlations between slip band locations and distinct features of the polycrystalline microstructure are observed in this material. Most of them are directly related to 3D aspects of the microstructure that are not discernable on the free surface.

\section{Slip Transmission in Textured Polycrystalline $\alpha$ Ti7Al}

Ti-7Al is a single-phase model material with HCP crystalline structure and composition similar to the HCP phase of Ti-6Al-4V. It is wrought processed and exhibits a yield strength of about $600 \mathrm{MPa}$ and limited work hardening. A sample of Ti7Al manufactured by the Air Force Research Laboratory ${ }^{27,40}$ was loaded monotonically in tension at room temperature. Its properties are summarized in Fig. 6. It exhibits a yield strength of $576 \mathrm{MPa}$ and Young's modulus of $104 \mathrm{GPa}$ (Fig. 6a) at an average grain size of $80 \mu \mathrm{m}$ (Fig. 6d,e). A strong texture is observed, with $((2 \overline{1} 10))$-oriented crystals preferentially aligned along the loading direction (Fig. 6b). These orientations are favorably oriented for prismatic slip. The material was strained in situ to a total macroscopic strain of $1 \%$, the point at which HR-DIC data were collected. A number of slip bands of varied intensity are visible on the strain map shown in Fig. 6. The dark horizontal lines visible in
Fig. $6 \mathrm{c}$ originate from the stitching process that is involved while collecting DIC data over large ROIs. The ROI consists of a $302 \mu \mathrm{m} \times 876 \mu \mathrm{m}$ surface area that contains 29 individual grains. It is highlighted with dashed lines in Fig. 6d and e.

The aligned strain map obtained from HR-DIC is shown in Fig. 7a, overlapped with the IPF colored EBSD map (with reference to the loading direction). The most intense slip bands are shown in the 3D rendering in Fig. 7b. Unlike the prior case study, limited correlation is observed between the microstructure features (triple junctions, quadruple points, etc.) and the location of the slip bands. Longrange slip transmission is observed across the whole ROI, as shown in Supplementary Material A. This is likely due to the strong texture and similar orientations of most grains across the gauge, which presents a case of easy prismatic slip transmission. ${ }^{41}$ This case of long-range transmission of plasticity is common in microtextured titanium alloys. ${ }^{42}$ However, the most interesting observation is that all transmitted slip bands connect to a single point on the free surface. This is particularly striking in Supplementary Material B. Several other examples are shown in Fig. 7c, across grains A-C and D-E, highlighted with black arrows. These slip transmission events are readily observable on the free surface, and it is worth mentioning that when looking at the $3 \mathrm{D}$ reconstruction of the slip bands, the point where slip transmission occurs tends to be at the surface. This suggests a possible surface effect driving the nucleation of transmitted bands. More specific analyses should be conducted to further investigate this aspect. Techniques able to probe slip band connection both on the surface and within the bulk, such as topo-tomography, ${ }^{43,} 44$

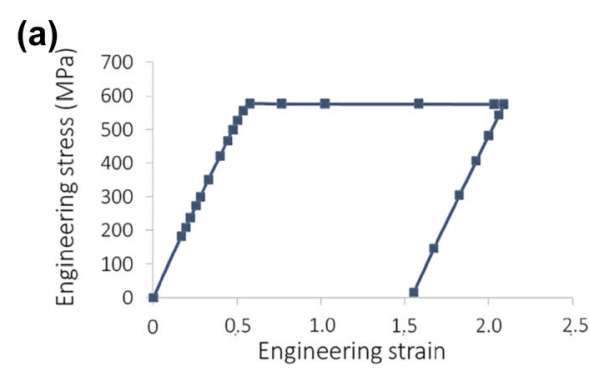

(b)

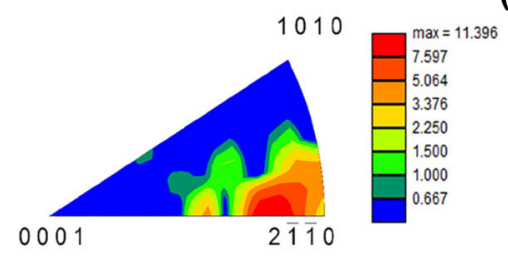

(c)

(d)

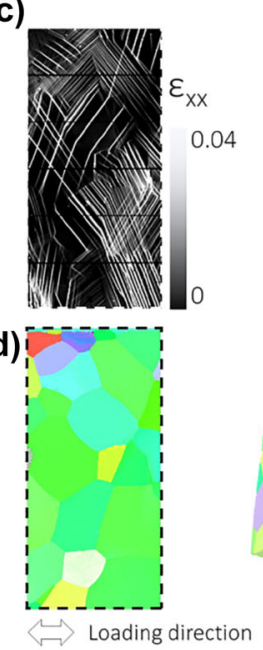

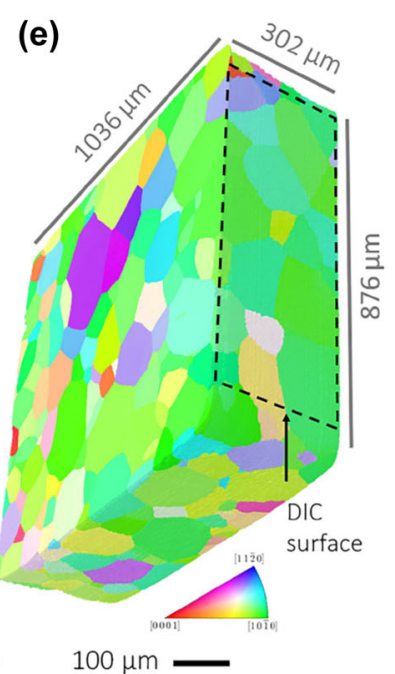

Fig. 6. Microstructure and properties of the Ti-7Al sample: (a) engineering stress-strain curve, (b) microtexture with respect to the loading direction and colored as a multiple of random distribution, (c) strain map obtained by DIC; the loading direction is horizontal, (d) corresponding EBSD map in IPF colors with respect to the loading direction and (e) 3D EBSD dataset in IPF colors with respect to the loading direction. The dashed rectangle corresponds to the overlapping region between the DIC and 3D EBSD datasets. 
(a)

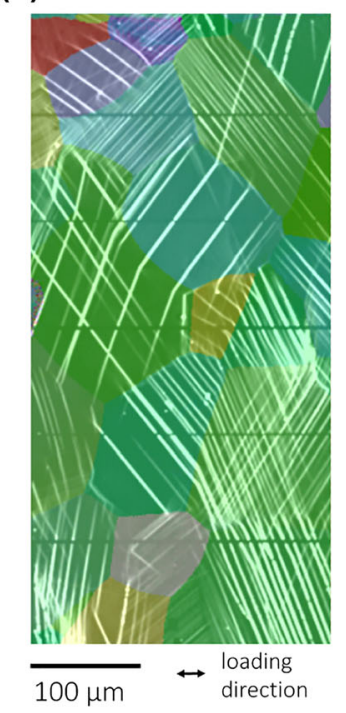

(b)

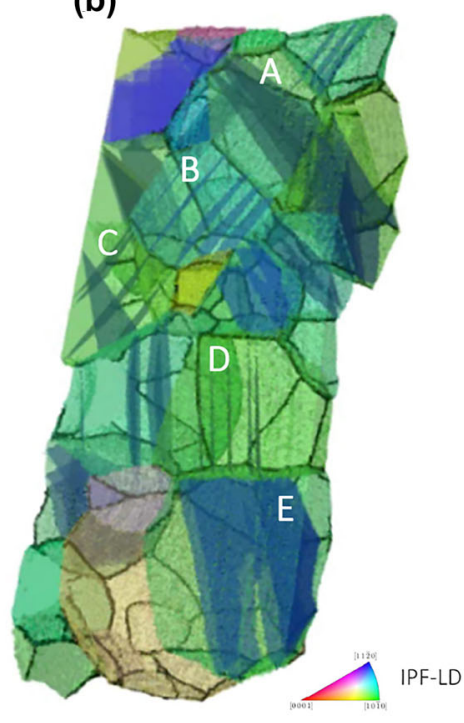

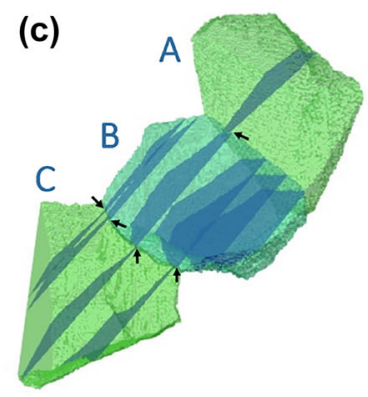

(c)

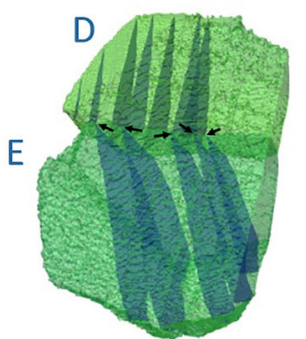

Fig. 7. Merged Ti7Al dataset: (a) HR-DIC strain map overlapped with the EBSD dataset, (b) 3D rendering of the 3D dataset with the slip bands of highest intensity added to the ROI, (c) two examples of prismatic slip transmission.

would certainly shed light on the configurations of slip transmission.

\section{DISCUSSION}

\section{Correlated Data Analysis Tools}

The present framework enables the reconstruction of slip bands in 3D, using the combination of $3 \mathrm{D}$ EBSD and HR-DIC. Correlative analysis of the location of slip bands in relation to the $3 \mathrm{D}$ microstructure can be conducted by observation of individual grains, either automatically or statistically, using custom Python or Matlab scripts. To do so, a number of tools have been developed and will be openly available in the near future in the form of a Python package called Argos. These tools include $2 \mathrm{D}$ and 3D segmentation and vectorization scripts, data alignment routines, merging procedures (functions that enable finding the grain to which a given slip band belongs, for example) as well as plotting scripts and functions.

\section{Limits of the Current Technique}

As HR-DICs are surface measurements, the merged datasets are limited to the slip bands in the first layer of grains, beneath the DIC surface. Such surface phenomena are particularly relevant to fatigue, and an improved understanding of fatigue crack initiation motivated the studies on Inconel 718. However, surface observations may have limitations. First, the slip bands may be influenced by the different states of stress at the surface. The various examples of slip transmission shown in Sect. Slip Transmission in Textured Polycrystalline $\alpha$ Ti7Al reveal that the transmission events observed by HR-DIC may be influenced by the free surface. Conversely, at temperatures where fatigue cracks initiate sub-surface in nickel alloys, the bands still form parallel to and slightly offset from twin boundaries. ${ }^{45}$

As mentioned in the introduction, various techniques can be employed to map either 3D microstructures or full $3 \mathrm{D}$ strain fields. While it possesses a good spatial resolution and the ability to analyze a wide range of materials systems, Tribeam tomography remains a destructive technique. While DIC can be collected at various macroscopic strains, the sample must be unloaded and prepared for Tribeam tomography. For example, a simultaneous study of lattice rotation and slip band formation cannot be performed in situ. While some lattice rotation can remain after unloading, dynamic correlative observations ${ }^{46}$ cannot be performed with the present set of tools. The multi-modal data merging framework can, however, be applied to alternative characterization techniques, as discussed further.

\section{Applicability to Other Types of Data}

The multi-modal data combination approach shown here for the Ti-7Al and Inconel 718 examples is generalizable to a wide range of spatially distributed measurements. The need for recognizable fiducials between the required data modalities is likely to persist unless noise and distortions that are inherent to each detector modality can be completely eliminated and/or perfectly simulated across all modalities. Certainly electron microscopy modality simulations have improved dramatically, ${ }^{47}$ making the multi-modal data fusion process much more straightforward, as evidenced by the indexing improvements from the dictionary and spherical 
approaches. ${ }^{20,34,48}$ However, multi-modal data fusion need not only be applied to electron imaging modalities. For instance both laboratory ${ }^{49-53}$ and synchrotron $x$-ray imaging modes ${ }^{54,55}$ have enjoyed continued diversification and enhancements leading to improved mapping of grain structure and crystallographic orientation compared to 3D EBSD methods. $^{56-58}$

\section{CONCLUSION, LIMITATIONS AND FUTURE WORK}

A framework for multi-modal data merging, involving the recombination of HR-DIC, 3D EBSD and $3 \mathrm{D}$ BSE images, is presented. It enables the automated reconstruction and visualization of hundreds of slip bands mapped over millimetric regions of interest, allowing statistical analyses using custom post-processing scripts. This enables automated, non-human biased analyses of the merged data. Whereas the illustration examples presented here involve the combination of $3 \mathrm{D}$ microstructure information with surface (slip traces) measurements, the framework can also be used for the recombination of slip bands imaged directly in the bulk of the material, if they exist. Furthermore, the 3D microstructure measurements need not be EBSD and can be extended to other 3D mapping techniques.

\section{ACKNOWLEDGEMENTS}

The work on Inconel 718 is funded by the US Department of Energy, Office of Basic Energy Sciences, Program DE-SC0018901. Use was made of computational facilities purchased with funds from the National Science Foundation (CNS-1725797) and administered by the Center for Scientific Computing (CSC). The CSC is supported by the California NanoSystems Institute and the Materials Research Science and Engineering Center (MRSEC; NSF DMR 1720256) at UC Santa Barbara.

\section{CONFLICT OF INTEREST}

The authors declare that they have no conflict of interest.

\section{SUPPLEMENTARY MATERIAL}

A. Merged Ti-7Al dataset B. Surface transmission in $\mathrm{Ti}-7 \mathrm{Al}$

\section{SUPPLEMENTARY INFORMATION}

The online version contains supplementary material available at https://doi.org/10.1007/s11837021-04894-6.

\section{REFERENCES}

1. C. Blochwitz, J. Brechbühl, W. Tirschler, Mater. Sci. Eng.: A 210(1), 42 (1996).

2. B. Simkin, B. Ng, T. Bieler, M. Crimp, D. Mason, Intermetallics 11(3), 215 (2003).

3. S. Fréchard, F. Martin, C. Clément, J. Cousty, Mater. Sci. Eng.: A 418(1), 312 (2006).

4. Z. Keshavarz, M.R. Barnett, Scripta Materialia 55(10), 915 (2006).

5. J.R. Seal, M.A. Crimp, T.R. Bieler, C.J. Boehlert, Mater. Sci. Eng.: A 552, 61 (2012).

6. J.R. Seal, T. Bieler, M. Crimp, B. Britton, A. Wilkinson, Microscopy and Microanalysis 18, 702 (2012). https://www. proquest.com/scholarly-journals/characterizing-slip-transfer -commercially-pure/docview/1270465065/se-2?accountid=14 522. Copyright - Copyright Microscopy Society of America 2012; Dernière mise à jour - 2015-08-15.

7. H. Li, D. Mason, T. Bieler, C. Boehlert, M. Crimp, Acta Materialia 61(20), 7555 (2013).

8. J. Stinville, M. Echlin, D. Texier, F. Bridier, P. Bocher, T. Pollock, Experimental Mechanics pp. 1-20 (2015). 10.1007/s11340-015-0083-4. http://dx.doi.org/10.1007/s1134 0-015-0083-4.

9. S. Hémery, P. Villechaise, Scripta Materialia130, 157 (2017).

10. C. Cepeda-Jiménez, C. Prado-Martínez, M. Pérez-Prado, Acta Materialia 145, 264 (2018).

11. S. Hémery, P. Villechaise, Acta Materialia 171, 261 (2019).

12. M.A. Charpagne, F. Strub, T.M. Pollock, Mater. Charact. 150, 184 (2019).

13. X. Xu, D. Lunt, R. Thomas, R.P. Babu, A. Harte, M. Atkinson, J. da Fonseca, M. Preuss, Acta Materialia 175, 376 (2019).

14. R. Alizadeh, M. Peña-Ortega, T. Bieler, J. LLorca, Scripta Materialia 178, 408 (2020). https://doi.org/10.1016/j.scriptamat.2019.12.010. https://www.sciencedirect.com/science/a rticle/pii/S1359646219307298.

15. H. Lim, J.D. Carroll, J.R. Michael, C.C. Battaile, S.R. Chen, J.M.D. Lane, Acta Materialia 185, 1 (2020).

16. R. Sperry, S. Han, Z. Chen, S.H. Daly, M.A. Crimp, D.T. Fullwood, Mater. Charact. 173, 110941 (2021).

17. A. Weidner, H. Biermann, Adv. Eng. Mater. 23(4), 2170011 (2021).

18. A.D. Kammers, S. Daly, Exp. Mech. 53(9), 1743 (2013).

19. J. Stinville, M. Echlin, D. Texier, F. Bridier, P. Bocher, T. Pollock, Exp. Mech. 56(2), 197 (2015).

20. Y.H. Chen, S.U. Park, D. Wei, G. Newstadt, M.A. Jackson, J.P. Simmons, M. De Graef, A.O. Hero, Microscopy Microanal. 21(03), 739 (2015).

21. J.C. Stinville, W.C. Lenthe, M.P. Echlin, P.G. Callahan, D. Texier, T.M. Pollock, Int. J. Fract. 208(1), 221 (2017).

22. L. Renversade, R. Quey, W. Ludwig, D. Menasche, S. Maddali, R.M. Suter, A. Borbély, IUCrJ 3(1), 32 (2016).

23. H.F. Poulsen, S.F. Nielsen, E.M. Lauridsen, S. Schmidt, R.M. Suter, U. Lienert, L. Margulies, T. Lorentzen, D. Juul Jensen, Journal of Applied Crystallography 34(6), 751 (2001). 10.1107/S0021889801014273. https://doi.org/10. 1107/S0021889801014273.

24. J. Rowenhorst, L. Nguyen, R.W. Fonda, Microscopy Microanal. 24(S1), 552-553 (2018). https://doi.org/10.1017/S1431 927618003252 .

25. M. Uchic, M. Groeber, M. Shah, P. Callahan, A. Shiveley, M. Scott, M. Chapman, J. Spowart, in Proceedings of the 1st International Conference on 3D Materials Science, ed. by M. De Graef, H.F. Poulsen, A. Lewis, J. Simmons, G. Spanos (Springer International Publishing, Cham, 2016), pp. 195-202.

26. M.P. Echlin, T.L. Burnett, A.T. Polonsky, T.M. Pollock, P.J. Withers, Curr. Opin. Solid State Mater. Sci. 24(2), 100817 (2020).

27. S.L. Semiatin, N.C. Levkulich, A.A. Salem, A.L. Pilchak, Metal. Mater. Trans. A 51(9), 4695 (2020). 
28. M. Charpagne, J. Stinville, P. Callahan, D. Texier, Z. Chen, P. Villechaise, V. Valle, T. Pollock, Mater. Charact. 163, 1 (2020).

29. A. Kammers, S. Daly, Exp. Mech. 53(8), 1333 (2013).

30. M.P. Echlin, M. Straw, S. Randolph, J. Filevich, T.M. Pollock, Mater. Charact. 100, 1 (2015).

31. W.C. Lenthe, J.C. Stinville, M.P. Echlin, Z. Chen, S. Daly, T.M. Pollock, Ultramicroscopy 195, 93 (2018).

32. F. Bourdin, J. Stinville, M. Echlin, P. Callahan, W. Lenthe, C. Torbet, D. Texier, F. Bridier, J. Cormier, P. Villechaise, T. Pollock, V. Valle, Acta Materialia 157, 307 (2018).

33. S.J. Randolph, J. Filevich, A. Botman, R. Gannon, C. Rue, M. Straw, J. Vac. Sci. Technol. B 36(6), 06JB01 (2018). h ttps://doi.org/10.1116/1.5047806

(doi.org/10.1116/ 1.5047806).

34. W. Lenthe, S. Singh, M.D. Graef, Ultramicroscopy 207, 112841 (2019).

35. M.A. Groeber, M.A. Jackson, Integr. Mater. Manuf. Innov $3(1), 56$ (2014).

36. G. Nolze, Ultramicroscopy 107(2-3), 172 (2007).

37. E. Holm, R. Cohn, N. Gao, Metall Mater. Trans. A 51, 5985 (2020). https://doi.org/10.1007/s11661-020-06008-.

38. J. Ahrens, B. Geveci, C. Law, The visualization handbook 717 (2005)

39. M. Charpagne, J. Hestroffer, A. Polonsky, M. Echlin, D. Texier, I. Beyerlein, T. Pollock, J. Stinville, Acta Materialia submitted, 0 (2021).

40. K. Chatterjee, A. Venkataraman, T. Garbaciak, J. Rotella, M. Sangid, A. Beaudoin, P. Kenesei, J.S. Park, A. Pilchak, Int. J. Solids Struct. 94-95, 35 (2016).

41. T. Kehagias, P. Komninou, G. Dimitrakopulos, J. Antonopoulos, T. Karakostas, Scripta Metallurgica et Materialia 33(12), 1883 (1995).

42. M.P. Echlin, J.C. Stinville, V.M. Miller, W.C. Lenthe, T.M. Pollock, Acta Mater. 114, 164 (2016).

43. W. Ludwig, S. Schmidt, E.M. Lauridsen, H.F. Poulsen, J. Appl. Crystal. 41(2), 302 (2008).

44. H. Proudhon, M. Pelerin, A. King, W. Ludwig, Curr. Opin. Solid State Mater. Sci. 24(4), 100834 (2020).

45. J.C. Stinville, P.G. Callahan, M.A. Charpagne, M.P. Echlin, V. Valle, T.M. Pollock, Acta Mater. 186, 172 (2020). https://d oi.org/10.1016/j.actamat.2019.12.009.
46. J. Stinville, T. Francis, A. Polonsky, Experimental Mechanics 61, 331-(2021). 10.1007/s11340-020-00632-2.

47. M.D. Graef, M. Jackson, Saransh, Wlenthe, J. Kleingers, S. Wright, Josephtessmer. Emsoft-org/emsoft: Release 4.2 to synchronize with di tutorial paper (2019). https://zenodo.or $\mathrm{g} / \mathrm{record} / 2581285$.

48. M.A. Jackson, E. Pascal, M.D. Graef, Integr. Mater. Manuf. Innov. 8(2), 226 (2019).

49. A. King, P. Reischig, J. Adrien, W. Ludwig, J. Appl. Crystal. 46(6), 1734 (2013)

50. S. McDonald, P. Reischig, C. Holzner, E. Lauridsen, P. Withers, A. Merkle, M. Feser, Sci. Rep. 5, 14665 (2015).

51. C. Holzner, L. Lavery, H. Bale, A. Merkle, S. McDonald, P. Withers, Y. Zhang, D.J. Jensen, M. Kimura, A. Lyckegaard et al., Microscopy Today 24(4), 34-43 (2016). https://doi.org/ 10.1017/S1551929516000584.

52. Y. Zhao, S. Niverty, X. Ma, X. Liu, N. Chawla, Mater. Charact. 170, 110716 (2020).

53. S.A. McDonald, T.L. Burnett, J. Donoghue, N. Gueninchault, H. Bale, C. Holzner, E.M. Lauridsen, P.J. Withers, Mater. Charact. 172, 110814 (2021).

54. H. Proudhon, J. Li, W. Ludwig, A. Roos, S. Forest, Adv. Eng. Mater. 19(8), 1600721 (2017).

55. M. Miller, D. Pagan, A. Beaudoin, Metall. Mater. Trans. A 51, 4360 (2020). https://doi.org/10.1007/s11661-020-05888w.

56. W.C. Lenthe, M.P. Echlin, A. Trenkle, M. Syha, P. Gumbsch, T.M. Pollock, J. Appl. Crystal. 48(4), 1034 (2015).

57. A. Trenkle, M. Syha, W. Rheinheimer, P. Callahan, L. Nguyen, W. Ludwig, W. Lenthe, M.P. Echlin, T.M. Pollock, D. Weygand, M.D. Graef, M.J. Hoffmann, P. Gumbsch, J. Appl. Crystal. 53(2), 349 (2020).

58. K. Louca, H. Abdolvand, Mater. Charact. 171, 110753 (2021).

Publisher's Note Springer Nature remains neutral with regard to jurisdictional claims in published maps and institutional affiliations. 